\title{
Coupled Dictionary Learning for Multi-contrast MRI Reconstruction
}

\author{
Pingfan Song, Student Member, IEEE, Lior Weizman, Member, IEEE, João F. C. Mota, Member, IEEE, \\ Yonina C. Eldar, Fellow, IEEE, and Miguel R. D. Rodrigues, Senior Member, IEEE
}

\begin{abstract}
Magnetic resonance (MR) imaging tasks often involve multiple contrasts, such as T1-weighted, T2-weighted and Fluid-attenuated inversion recovery (FLAIR) data. These contrasts capture information associated with the same underlying anatomy and thus exhibit similarities in either structure level or gray level. In this paper, we propose a Coupled Dictionary Learning based multi-contrast MRI reconstruction (CDLMRI) approach to leverage the dependency correlation between different contrasts for guided or joint reconstruction from their undersampled $k$-space data. Our approach iterates between three stages: coupled dictionary learning, coupled sparse denoising, and enforcing $k$-space consistency. The first stage learns a set of dictionaries that not only are adaptive to the contrasts, but also capture correlations among multiple contrasts in a sparse transform domain. By capitalizing on the learned dictionaries, the second stage performs coupled sparse coding to remove the aliasing and noise in the corrupted contrasts. The third stage enforces consistency between the denoised contrasts and the measurements in the $k$-space domain. Numerical experiments, consisting of retrospective under-sampling of various MRI contrasts with a variety of sampling schemes, demonstrate that CDLMRI is capable of capturing structural dependencies between different contrasts. The learned priors indicate notable advantages in multi-contrast MR imaging and promising applications in quantitative MR imaging such as MR fingerprinting.
\end{abstract}

Index Terms-multi-contrast MRI, coupled dictionary learning, sparse coding, joint reconstruction, MR fingerprinting

\section{INTRODUCTION}

Magnetic Resonance Imaging (MRI) is a noninvasive and non-ionizing medical imaging technique widely used for medical diagnosis, clinical analysis, and staging of disease. Different MRI pulse sequences produce exquisite images of body parts with different contrasts, such as T1-weighted, T2weighted and fluid-attenuated inversion recovery (FLAIR),

This work was supported by EPSRC grant EP/K033166/1, by the Royal Society International Exchange Scheme IE160348, by the European Union's Horizon 2020 grant ERC-BNYQ, by the Israel Science Foundation grant no. 335/14, by ICore: the Israeli Excellence Center 'Circle of Light', by the Ministry of Science and Technology, Israel, and by UCL ORS Scholarship.

Pingfan Song was with the Department of Electronic \& Electrical Engineering, University College London, and at present is with the Dept. of EEE, Imperial College London, London SW7 2AZ, UK. (e-mail: p.song@imperial.ac.uk)

Lior Weizman was with the Department of Electrical Engineering, Technion - Israel Institute of Technology, Haifa 32000, Israel. (e-mail: weizmanl@tx.technion.ac.il)

João F. C. Mota is with the Department of School of Engineering \& Physical Sciences, Heriot-Watt University, Edinburgh EH14 4AS, UK. (email: j.mota@hw.ac.uk)

Yonina C. Eldar is with the Faculty of Math \& CS, Weizmann institute of Science, Rehovot 7610001, Israel. (e-mail: yonina.eldar@weizmann.ac.il)

Miguel R. D. Rodrigues is with the Department of Electronic \& Electrical Engineering, University College London, London WC1E 6BT, UK. (e-mail: m.rodrigues@ucl.ac.uk)

Code and data is available on https://github.com/pingfansong/CDLMRI enabling outstanding visualization of anatomical structures. However, MRI physics and physiological constraints make the MRI scanning procedure time-consuming. The relatively slow imaging speed of MRI affects clinical throughput and also leads to patients' discomfort. Therefore, a variety of MRI techniques have been proposed to shorten acquisition time via reducing the amount of data required for accurate reconstruction.

Single-contrast Reconstruction. MRI reconstruction from under-sampled measurements has been thoroughly investigated in the case of single contrast acquisition [1]-[3]. The pioneering framework proposed by Lustig et al. [1], motivated by compressive sensing theory [4]-[7], uses the fact that MR images are sampled in the spatial frequency domain (a.k.a. $k$-space) and can be represented as a sparse combination of fixed, predefined bases, for example, wavelets. See also further developments in [8]-[12]. Ravishankar et al. [2] proposed a dictionary learning approach for MRI reconstruction, DLMRI, based on the fact that patches of MR images can be sparsely represented with respect to a set of learned adaptive bases [13]. These learned bases are one of the reasons why DLMRI outperforms SparseMRI [1]. Caballero et al. [3] apply dictionary learning to spatio-temporal 3D dynamic complex-valued cardiac cine MRI data via a sparsity model that combines both spatial sparsity (for patches) and time sparsity (for temporal gradients), and treats the real and imaginary parts of the dataset independently. Recently, several deep learning based singlecontrast MRI reconstruction approaches were proposed [14][18]. For example, Sun et al. [14] propose a deep ADMM-net defined over a data flow graph that is derived from the iterative procedures in the Alternating Direction Method of Multipliers (ADMM) algorithm for optimizing a CS-based MRI model.

Multi-contrast Reconstruction. Multi-contrast MR imaging is attracting increasing amount of attention and being investigated in various scenarios [19]-[31]. Considering that each contrast depicts different physical properties of the same underlying anatomy and tissue, different image contrasts are highly correlated [21], [32], [33]. Such correlation has been exploited to shorten acquisition time or reduce the amount of measurements mainly in two scenarios: 1) guided reconstruction - the under-sampled target contrast is reconstructed with the aid of another fully-sampled contrast as guidance/reference, and 2) joint reconstruction - both contrasts are under-sampled and jointly reconstructed.

For the scenario where the target contrast is under-sampled and the reference is fully sampled, Weizman et al. [19], [20] proposed reference-based MRI (RefMRI), which exploits 


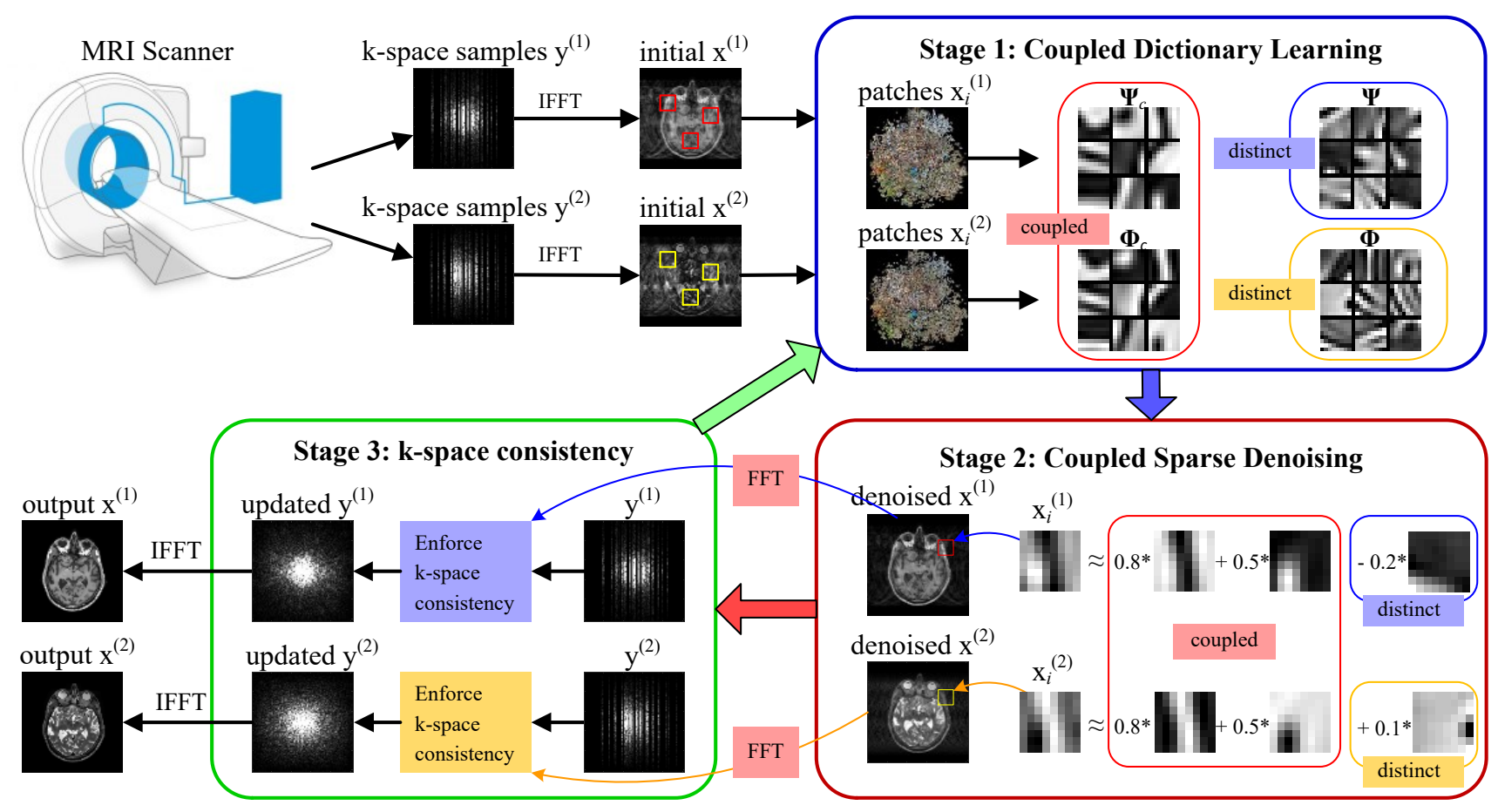

Figure 1: Coupled Dictionary Learning for multi-contrast MRI reconstruction framework. It reconstructs the contrasts $\mathbf{x}^{(1)}$ and $\mathbf{x}^{(2)}$ jointly from their $k$-space samples. CDLMRI iterates between three stages - coupled dictionary learning, coupled sparse denoising and $k$-space consistency - to progressively remove aliasing and noise and recover fine details.

the similarity between different contrasts at the pixel level (e.g., T2-weighted and FLAIR). Namely, it reconstructs the target contrast given the guidance contrast as reference. Their approach is specific to contrasts with gray-level similarity, not structural similarity, which is our focus here. Ehrhardt et al. [21] proposed an approach that uses structure-guided total variation (STV) to extract the location and direction priors from a guidance contrast to aid the reconstruction of the target contrast.

For the scenario where both contrasts are under-sampled, Bilgic et al. [22] proposed a joint Bayesian compressed sensing based algorithm to exploit the similarity of spatial derivatives/gradients in multi-contrast MRI for joint reconstruction. $\mathrm{Qu}$ et al. [23] suggested a patch-based nonlocal operator (PANO) to make use of the similarity within and between multi-contrast MRI patches in a CS framework. By combining joint total variation (JTV) and group wavelet-sparsity regularization, Huang et al. [24] suggested a Fast Joint-Gradient Projection (FJGP) to simultaneously reconstruct multiple MRI contrasts.

Our method. It is known that different contrasts from the same anatomy exhibit complex relationships, including both similarities and disparities in gray level and structure level. Capturing these complex relationships is challenging for compressive sensing based methods due to limited adaptivity of predefined bases. In addition, most existing multi-contrast MRI reconstruction methods are unable to capture differences between distinct image modalities associated with the same phenomenon, and thus tend to introduce noticeable texturecopying artifacts [19]-[24].
In order to address these issues, we propose a new multicontrast MRI reconstruction approach that is able to capture complex relationships between different contrasts both in gray level (e.g., FLAIR and T2-weighted, as in [19]) and structurelevel (e.g., T1-weighted and T2-weighted). The proposed multi-contrast MRI reconstruction framework consists of three stages: coupled dictionary learning, coupled sparse denoising and enforcing $k$-space consistency, as shown in Fig. 1. The first stage learns a set of dictionaries with the goal of capturing similarities and disparities of textures, edges, boundaries, or other salient primitives, across different contrasts. The second stage performs coupled sparse coding to remove aliasing and noise in the corrupted contrasts, by capitalizing on the learned adaptive dictionaries. The third stage enforces consistency between the denoised contrasts and the measurements in the $k$-space domain. Comprehensive experiments demonstrate that our approach outperforms representative methods in both guided and joint reconstruction with better visual quality and peak signal-to-noise ratio (PSNR) performance. These benefits, however, are at the expense of increased computational complexity and memory requirements with respect to methods that have no learning stage.

Our contributions can be summarised as follows:

- We propose a framework for reconstructing multi-contrast MRI based on coupled dictionary learning.

- We also suggest a learning algorithm to learn the required dictionaries, so that cross-modality dependency correlation, including both similarities and disparities, is expressed using joint sparse representations. 
- A refined sparse coding operation is then designed to accelerate and improve coupled sparse denoising.

- Finally, we evaluate the proposed algorithm on a variety of MRI contrasts and various sampling patterns for both guided and joint reconstruction.

Organization. In Section II, we present the MRI reconstruction problem and also introduce some background and related work. In Section III, we introduce our data model for multi-contrast MR images and the proposed CDLMRI approach. Section IV is devoted to experiments. We conclude in Section VI.

\section{BACKGROUND AND RELATED WORK}

We start by formalising the case of single-constrast MRI. Let $\mathbf{x}^{(1)} \in \mathcal{C}^{N}$ denote the vectorized 2D MRI contrast of size $\sqrt{N} \times \sqrt{N}$, and $\mathbf{y}^{(1)}=\mathbf{F}_{u 1} \mathbf{x}^{(1)} \in \mathcal{C}^{m_{1}}\left(m_{1}<N\right)$ denote the under-sampled $k$-space measurements of $\mathbf{x}^{(1)}$, with $\mathbf{F}_{u 1} \in \mathcal{C}^{m_{1} \times N}$ the corresponding under-sampled Fourier transform matrix, i.e. a partial 2D DFT matrix. We refer to the problem of reconstructing $\mathbf{x}^{(1)}$ from $\mathbf{y}^{(1)}$ single-contrast MRI reconstruction.

In many scenarios, we also have access to an additional contrast $\mathbf{x}^{(2)} \in \mathcal{C}^{N}$ associated with the same underlying anatomy. The corresponding vector of $k$-space samples is $\mathbf{y}^{(2)}=\mathbf{F}_{u 2} \mathbf{x}^{(2)} \in \mathcal{C}^{m_{2}}$, where $\mathbf{F}_{u 2} \in \mathcal{C}^{m_{2} \times N}$ is the under-sampled or fully-sampled Fourier transform matrix. We distinguish between two cases: (1) If the additional contrast $\mathbf{x}^{(2)}$ is fully-sampled, i.e. $m_{2}=N$, the reconstruction of $\mathbf{x}^{(1)}$ from $\mathbf{y}^{(1)}$, given $\mathbf{x}^{(2)}$ for guidance/reference, is called guided or reference-based reconstruction. (2) If $\mathbf{x}^{(2)}$ is also undersampled, i.e. $m_{2}<N$, the problem of reconstructing $\mathbf{x}^{(1)}$ and $\mathbf{x}^{(2)}$ from $\mathbf{y}^{(1)}$ and $\mathbf{y}^{(2)}$ is referred to as joint reconstruction.

Next, we briefly introduce several representative types of single-contrast and multi-contrast MRI reconstruction approaches. Whenever we refer to single-contrast MRI reconstruction, we omit superscript ${ }^{(1)}$ for notational simplicity.

\section{A. Compressive Sensing based Single MRI Reconstruction}

The reconstruction of $\mathbf{x}$ from $\mathbf{y}$ is an ill-posed problem with an infinite number of solutions, due to the insufficient number of observations. To find a suitable solution, Compressive Sensing based single-contrast MRI (CSMRI) reconstruction exploits sparsity to regularize this ill-posed problem. Based on the fact that a MR image can be represented as a sparse combination of fixed, predefined bases, it is possible to recover the desired contrast from its measurements as follows:

$$
\underset{\mathbf{x}}{\operatorname{minimize}}\left\|\mathbf{F}_{u} \mathbf{x}-\mathbf{y}\right\|_{2}^{2}+\lambda\|\mathbf{\Psi} \mathbf{x}\|_{1}+\gamma\|\mathbf{x}\|_{T V}
$$

where $\|\cdot\|_{1}$ denotes the $\ell_{1}$ norm of a vector, i.e., the sum of the absolute values of the entries in a vector, and $\|\mathbf{x}\|_{T V}=$ $\sum_{i=1}^{N} \sqrt{\left(\nabla_{1} \mathbf{x}_{i}\right)^{2}+\left(\nabla_{2} \mathbf{x}_{i}\right)^{2}}$ is the total variation, where $\nabla_{1}$ and $\nabla_{2}$ denote the forward finite difference operators on the first and second coordinates. The parameters $\lambda>0$ and $\gamma>$ 0 trade off sparsity, piece-wise smoothness and measurement fidelity. Commonly, $\boldsymbol{\Psi}$ is a fixed matrix, for example, one that implements the discrete Fourier transform (DFT), wavelet, or contourlet transforms. There exists a variety of algorithms to solve such problems, e.g. [1], [8]-[10], [23], [34], [35].

\section{B. Dictionary Learning based Single MRI Reconstruction}

Compared with a pre-defined dictionary used in CSMRI, an adaptive dictionary is able to further promote sparsity of the signal and hence potentially require fewer samples for successful reconstruction [2], [36]. DLMRI by Ravishankar and Bresler [2] integrates dictionary learning with compressive sensing to achieve more effective MRI reconstruction. Specifically, [2] attempts to solve

$$
\begin{array}{cl}
\min _{\mathbf{x}, \mathbf{D}, \boldsymbol{\alpha}_{i j}} & \sum_{i j}\left\|\mathbf{R}_{i j} \mathbf{x}-\mathbf{D} \boldsymbol{\alpha}_{i j}\right\|_{2}^{2}+\nu\left\|\mathbf{F}_{u} \mathbf{x}-\mathbf{y}\right\|_{2}^{2} \\
\text { s.t. } & \left\|\boldsymbol{\alpha}_{i j}\right\|_{0} \leq s \quad \forall i, j,
\end{array}
$$

where $\mathbf{R}_{i j}$ is the operator that extracts a patch with top-left corner at the location $(i, j)$ of $\mathbf{x}, \mathbf{D}$ denotes the dictionary to be learned, $\boldsymbol{\alpha}_{i j}$ denotes the sparse representation of the patch $\mathbf{R}_{i j} \mathbf{x}$ with respect to $\mathbf{D}$, and $s$ is the sparsity constraint. Formulation (2) involves an assumption that each image patch admits a sparse representation $\boldsymbol{\alpha}_{i j}$ with respect to the learned dictionary $\mathbf{D}$, i.e., $\mathbf{R}_{i j} \mathbf{x}=\mathbf{D} \boldsymbol{\alpha}_{i j}$.

The first term in the cost function represents the quality of the sparse approximation of the image patches and the second term enforces data fidelity in $k$-space. Therefore, DLMRI iterates between two steps: (1) performing dictionary learning and sparse coding in the image space to update the first term, and (2) performing data consistency in the $k$-space to update the second term. The quality of the reconstructed MRI increases along with iterations. Our framework is inspired by this algorithm, but we generalize it to guided or joint reconstruction for multi-contrast MRI. In addition, we also propose a different learning algorithm, making improvements on the training strategy, speed and accuracy.

\section{Guided Reconstruction}

In the scenario in which a fully-sampled additional contrast $\mathbf{x}^{(2)}$ is available, Ehrhardt et al.'s STVMRI [21] exploits the location and direction priors from the guidance contrast $\mathbf{x}^{(2)}$ to aid in reconstruction of the target contrast $\mathbf{x}^{(1)}$ by introducing a structure-guided total variation ${ }^{1}\|\mathbf{x}\|_{S T V}$. STVMRI modifies the standard total variation regularization by introducing anisotropic weights derived from the guidance contrast $\mathrm{x}^{(2)}$, by solving

$$
\underset{\mathbf{x}^{(1)}}{\operatorname{minimize}}\left\|\mathbf{F}_{u 1} \mathbf{x}^{(1)}-\mathbf{y}^{(1)}\right\|_{2}^{2}+\gamma\left\|\mathbf{x}^{(1)}\right\|_{S T V},
$$

where, the parameter $\gamma>0$ trades off piece-wise smoothness and measurement fidelity.

Weizman et al. proposed a re-weighted approach, RefMRI [19] that solves a sequence of optimization problems to reconstruct the target contrast:

$$
\min _{\mathbf{x}^{(1)}}\left\|\mathbf{A}\left(\mathbf{F}_{u} \mathbf{x}^{(1)}-\mathbf{y}^{(1)}\right)\right\|_{2}^{2}+\lambda_{1}\left\|\mathbf{W}_{1} \mathbf{\Psi}_{\mathbf{x}^{(1)}}\right\|_{1}+\lambda_{2}\left\|\mathbf{W}_{2}\left(\mathbf{x}^{(1)}-\mathbf{x}^{(2)}\right)\right\|_{1}
$$

where the fully-sampled contrast $\mathbf{x}^{(2)}$ acts as reference, and A is a diagonal matrix that controls the weight given to the fidelity of certain measurements. The diagonal matrix $\mathbf{W}_{1}$

${ }^{1}\left\|\mathbf{x}^{(1)}\right\|_{S T V}=\sum_{i=1}^{N}\left|\mathbf{D}_{i} \nabla \mathbf{x}_{i}^{(1)}\right|$, where $\nabla$ denotes the forward finite difference operator, and $\mathbf{D}_{i}=\mathbf{I}-\boldsymbol{\xi}_{i} \boldsymbol{\xi}_{i}^{*}$ denotes directional weights matrix with $\boldsymbol{\xi}_{i}:=\nabla \mathbf{x}_{i}^{(2)} / \sqrt{\left(\nabla \mathbf{x}_{i}^{(2)}\right)^{2}+\eta^{2}}$ for $\eta>0$. The matrix field $\mathbf{D}_{i}$ is anisotropic as it has principal directions along and orthogonal to $\boldsymbol{\xi}_{i}$. 
weighs specific wavelet atoms in the reconstruction process and $\mathbf{W}_{2}$ weighs image regions according to their similarity level with the reference scan. The matrices $\mathbf{W}_{1}$ and $\mathbf{W}_{2}$ are re-adjusted recursively. Parameters $\lambda_{1}>0$ and $\lambda_{2}>0$ trade off self-sparsity, relative sparsity, and measurement fidelity.

\section{Joint Reconstruction}

When both contrasts $\mathbf{x}^{(1)}$ and $\mathbf{x}^{(2)}$ are under-sampled, joint reconstruction from their $k$-space measurements can be achieved by using Huang et al.'s Fast Joint-Gradient Projection (FJGP) algorithm [24] which solves:

$$
\min _{\mathbf{X}} \frac{1}{2} \sum_{s=1}^{2}\left\|\mathbf{F}_{u s} \mathbf{X}_{s}-\mathbf{y}_{s}\right\|_{2}^{2}+\lambda_{1}\|\mathbf{X}\|_{J T V}+\lambda_{2}\|\mathbf{\Psi} \mathbf{X}\|_{2,1},
$$

where $\mathbf{X}=\left[\mathbf{x}^{(1)}, \mathbf{x}^{(2)}\right]$ is a set of multi-contrast MR images. Here, $\mathbf{F}_{u s}$ and $\mathbf{y}_{s}$ are the sampling matrix and the $k$-space measurement vector for the $s$-th image, respectively. The joint total variation $\|\mathbf{X}\|_{J T V}=$ $\sum_{i=1}^{N} \sqrt{\sum_{s=1}^{T}\left(\left(\nabla_{1} \mathbf{X}_{i s}\right)^{2}+\left(\nabla_{2} \mathbf{X}_{i s}\right)^{2}\right)}$ encourages piecewise smooth areas for all the contrasts. The $\ell_{2,1}$ norm $\|\cdot\|_{2,1}$ represents the sum of the $\ell_{2}$ norms of the rows of a matrix, inducing group sparsity across different contrasts.

Most of these methods have limited capability of the capturing complex correlation between different contrasts and making full use of both gray level and structure level similarities. In addition, they tend to be susceptible to disparities between different contrasts. This motivates us to design a new learningbased robust multi-contrast MRI reconstruction approach for both guided and joint reconstruction scenarios.

\section{CDLMRI FORMULATION AND ALGORITHM}

In this section, we introduce the proposed data model for multi-contrast MR images, and then present the algorithm for performing either guided or joint multi-contrast MRI reconstruction.

Before diving into detailed formulations, we provide a brief introduction to the motivation and intuition underlying our approach. We highlight that our coupled data model is motivated by a common observation that different contrasts are often captured from the same underlying anatomy and thus exhibit inherent common characteristics. In particular, they often exhibit dependency correlations, including a variety of structure similarities, such as edges, textures, corners, and other salient primitives. For example, such dependencies can be found in a pair of T1-weighted and T2-weighted MRI, shown in Fig. 2 (a) and (c). If a first-order differential operator is applied on such a pair of contrasts, the resulting gradient maps exhibit resemblance, shown in Fig. 2 (b). The differential operation can be regarded as a transform which reveals similarities. To reveal and capture more complex dependencies among different image modalities, more sophisticated transforming techniques are needed. We thus propose to learn adaptive transforms via coupled dictionary learning. Intuitively, when different contrasts are split into pairs of small patches, their complex dependencies are disseminated into a variety of simpler and more concise local patterns that can be effectively captured by learned coupled dictionaries, shown in Fig. 2

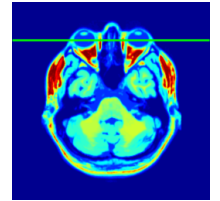

(a) T1w (left) and T2w (right) MRI

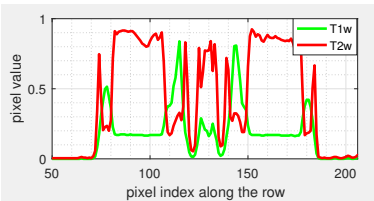

(c) pixel difference in a row of $\mathrm{T} 1 \mathrm{w} / \mathrm{T} 2 \mathrm{w}$

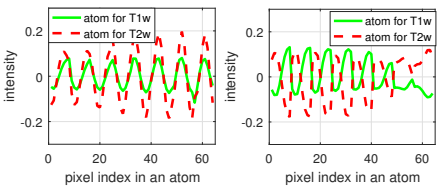

(e) some coupled atom pairs which are vectorized to 1D for illustration

Figure 2: Different contrasts often exhibit complex dependencies, sharing a variety of pixel and structure similarities, as shown in (a) and (c). (b) The gradient maps resulting from 1 -order differential show resemblance and thus may be used to capture the correlation, but it is not an effective way. (d-e) When splitting different contrasts into small patches, the complex dependencies are disseminated into a variety of simpler and more concise local patterns that can be effectively captured by learned coupled dictionaries.

(d-e). For any pair of coupled atoms, the intensity changing directions reflect their positive or negative correlation, while the absolute magnitudes capture their dependency correlation quantitatively. Consequently, the learned priors facilitates the leverage of correlation between different image modalities in the reconstruction task.

\section{A. Data Model for Multi-contrast MRI Data}

To utilize the dependency correlation between two MRI contrasts $\mathbf{x}^{(1)}$ and $\mathbf{x}^{(2)}$, we first propose a data model that captures their structural similarities and disparities. Our data model works with image patches, instead of entire images, because this allows modeling local image features effectively, as has been shown in other applications such as image denoising, super-resolution, inpainting, deblurring and demosaicing [37]. A more detailed analysis of the model can be found in Appendix C Section A.

Let $\mathbf{x}_{i j}^{(1)} \in \mathcal{C}^{n}$ and $\mathbf{x}_{i j}^{(2)} \in \mathcal{C}^{n}$ denote the vector representations of image patch pairs of size $\sqrt{n} \times \sqrt{n}$ extracted from the image $\mathbf{x}^{(1)} \in \mathcal{C}^{N}$ and $\mathbf{x}^{(2)} \in \mathcal{C}^{N}$, respectively, where the tuple $(i, j)$ denotes the coordinates of the top-left corner of the patches within the images. Formally, we write $\mathbf{x}_{i j}^{(1)}=\mathbf{R}_{i j} \mathbf{x}^{(1)}$ (resp. $\mathbf{x}_{i j}^{(2)}=\mathbf{R}_{i j} \mathbf{x}^{(2)}$ ), where the matrix $\mathbf{R}_{i j} \in \mathbb{R}^{n \times N}$ denotes the operator that extracts patch $\mathbf{x}_{i j}^{(1)}\left(\right.$ resp. $\mathbf{x}_{i j}^{(2)}$ ) from $\mathbf{x}^{(1)}$ (resp. $\mathbf{x}^{(2)}$ ). In order to capture both the similarities and the disparities between two different contrasts, we propose to associate each pair of patches $\left(\mathbf{x}_{i j}^{(1)}, \mathbf{x}_{i j}^{(2)}\right)$ with two types of sparse representations: a common sparse component that is 
shared by both contrasts, and a distinct sparse component for each contrast. This leads to the decomposition:

$$
\begin{aligned}
& \mathbf{x}_{i j}^{(1)}=\boldsymbol{\Psi}_{c} \mathbf{z}_{i j}+\boldsymbol{\Psi} \mathbf{u}_{i j}, \\
& \mathbf{x}_{i j}^{(2)}=\boldsymbol{\Phi}_{c} \mathbf{z}_{i j}+\boldsymbol{\Phi} \mathbf{v}_{i j},
\end{aligned}
$$

where $\mathbf{z}_{i j} \in \mathcal{C}^{K}$ is the common sparse representation shared by both contrasts, $\mathbf{u}_{i j} \in \mathcal{C}^{K}$ is a sparse component specific to contrast $\mathbf{x}^{(1)}$, and $\mathbf{v}_{i j} \in \mathcal{C}^{K}$ is a sparse component specific to contrast $\mathbf{x}^{(2)}$. In addition, $\boldsymbol{\Psi}_{c}=\left[\boldsymbol{\psi}_{c 1}, \cdots, \boldsymbol{\psi}_{c K}\right] \in \mathcal{C}^{n \times K}$ and $\boldsymbol{\Phi}_{c}=\left[\boldsymbol{\phi}_{c 1}, \cdots, \phi_{c K}\right] \in \mathcal{C}^{n \times K}$ are a pair of coupled dictionaries associated with the common sparse representation $\mathbf{z}_{i j}$, whereas $\boldsymbol{\Psi}=\left[\boldsymbol{\psi}_{1}, \cdots, \boldsymbol{\psi}_{K}\right] \in \mathcal{C}^{n \times K}$ and $\boldsymbol{\Phi}=$ $\left[\phi_{1}, \cdots, \phi_{K}\right] \in \mathcal{C}^{n \times K}$ are dictionaries associated with the distinct sparse representations $\mathbf{u}_{i j}$ and $\mathbf{v}_{i j}$, respectively. This model can be easily generalized for $\boldsymbol{\Psi}$ and $\boldsymbol{\Phi}$ to have a different number of atoms.

The data model such as (5) and (5) have also been successfully used to address multi-modal image super-resolution problems in [38]. Here, we extend them to multi-contrast MRI reconstruction. In particular, we propose a coupled dictionary learning algorithm, and a coupled sparse coding algorithm, which are different from [38]. (More detailed elaboration can be found in the Appendix C Section D.)

\section{B. CDLMRI}

We now present our approach for solving guided and joint multi-contrast MRI reconstruction problems leveraging the model (5) and (6). These two problems can be addressed by solving:

$$
\begin{aligned}
\min _{\substack{\mathbf{x}^{(1)}, \boldsymbol{\alpha} \\
\mathbf{x}^{(2)}, \mathbf{D}}} & \sum_{i j}\left\|\mathbf{R}_{i j} \mathbf{x}^{(1)}-\left(\boldsymbol{\Psi}_{c} \mathbf{z}_{i j}+\boldsymbol{\Psi} \mathbf{u}_{i j}\right)\right\|_{2}^{2}+\nu_{1}\left\|\mathbf{F}_{u 1} \mathbf{x}^{(1)}-\mathbf{y}^{(1)}\right\|_{2}^{2} \\
& +\sum_{i j}\left\|\mathbf{R}_{i j} \mathbf{x}^{(2)}-\left(\boldsymbol{\Phi}_{c} \mathbf{z}_{i j}+\boldsymbol{\Phi} \mathbf{v}_{i j}\right)\right\|_{2}^{2}+\nu_{2}\left\|\mathbf{F}_{u 2} \mathbf{x}^{(2)}-\mathbf{y}^{(2)}\right\|_{2}^{2} \\
\text { s.t. } \quad\left\|\mathbf{z}_{i j}\right\|_{0} \leq s_{c},\left\|\mathbf{u}_{i j}\right\|_{0} \leq s_{1},\left\|\mathbf{v}_{i j}\right\|_{0} \leq s_{2}, \forall i, j, & \\
& \left\|\left[\begin{array}{l}
\boldsymbol{\psi}_{c k} \\
\boldsymbol{\phi}_{c k}
\end{array}\right]\right\|_{2}^{2} \leq 1,\left\|\boldsymbol{\psi}_{k}\right\|_{2}^{2} \leq 1,\left\|\boldsymbol{\phi}_{k}\right\|_{2}^{2} \leq 1, \forall k,
\end{aligned}
$$

where $\boldsymbol{\alpha}$ denotes all the sparse representations $\mathbf{z}_{i j}, \mathbf{u}_{i j}, \mathbf{v}_{i j}$ and $\mathbf{D}$ denotes the four dictionaries $\boldsymbol{\Phi}_{c}, \boldsymbol{\Phi}, \boldsymbol{\Psi}_{c}, \boldsymbol{\Psi}$. The first and third terms in the objective ensure that the image patches are consistent with their postulated model (5)-(6). The second and fourth terms enforce that the target and guidance contrasts are consistent with their $k$-space measurements. For guided reconstruction, the fourth term and the variable $\mathbf{x}^{(2)}$ are fixed. The parameters $\nu_{1}=\lambda / \sigma_{1}$ and $\nu_{2}=\lambda / \sigma_{2}$ depend on the standard deviation of the measurement noise $\sigma_{1}$ and $\sigma_{2}$ and a positive constant $\lambda$. They are used to balance fidelity between the model and the measurements. In regard to the constraints, the first set of constraints imposes sparsity on the vectors $\mathbf{z}_{i j}, \mathbf{u}_{i j}, \mathbf{v}_{i j}$, while the second set of constraints normalizes the atoms of the dictionaries in order to remove the scaling ambiguity and avoid trivial solutions. This prevents the $\ell_{2}$ norm of the atoms from becoming infinitely large, which would make the sparsity constraint ineffective. The rationale for the $\ell_{2}$ norm constraints for each atom pair $\left[\psi_{c k} ; \phi_{c k}\right]$ is further described in Appendix A.

Problem (7) is nonconvex as both the dictionaries and the coefficients are optimization variables, and the $\ell_{0}$ pseudo-norm

\section{Algorithm 1 CDLMRI Algorithm}

Input: Under-sampled $k$-space measurements: $\mathbf{y}^{(1)}$ and $\mathbf{y}^{(2)}$;

Fidelity parameters: $\nu_{1}$ and $\nu_{2}$; Sparse constraints: $s_{c}, s_{1}$, $s_{2}$; Number of inner iterations for CDL: $L$; Number of outer iterations for CDLMRI: $T$; Number of overlapping patches at each pixel: $\beta$.

Output: Estimated $\tilde{\mathbf{x}}^{(1)}$ and $\tilde{\mathbf{x}}^{(2)}$.

Initialization: Initialize $\mathbf{x}^{(1)}$ and $\mathbf{x}^{(2)}$ as $\mathbf{x}^{(1)}=\mathbf{F}_{u 1}^{H} \mathbf{y}^{(1)}$ and $\mathbf{x}^{(2)}=\mathbf{F}_{u 2}^{H} \mathbf{y}^{(2)}$, respectively.

\section{Optimization:}

\section{1: for $t=1, \cdots, T$ do}

2: Stage 1) Coupled Dictionary Learning. Estimate the coupled dictionaries $\boldsymbol{\Psi}_{c}, \boldsymbol{\Psi}, \boldsymbol{\Phi}_{c}, \boldsymbol{\Phi}$ using Algorithm 2 .

3: Stage 2) Coupled Sparse Denoising. Estimate the sparse representations $\mathbf{z}_{i j}, \mathbf{u}_{i j}$ and $\mathbf{v}_{i j}$ by solving (10), (11) and (12) using the OMP algorithm, which leads to denoised patches $\hat{\mathbf{x}}_{i j}^{(1)}$ and $\hat{\mathbf{x}}_{i j}^{(2)}$.

4: Stage 3) $k$-space Consistency. Enforce data consistency with $k$-space measurements to estimate $\tilde{\mathbf{x}}^{(1)}$ and $\tilde{\mathbf{x}}^{(2)}$ as in (15).

5: end for

constraints are nonconvex. Therefore, we attempt to solve this problem by iterating between three stages: 1) Coupled dictionary learning, 2) Coupled sparse denoising, and 3) Enforcing $k$-space consistency, as shown in Algorithm 1. In Stage 1, we perform coupled dictionary learning on a subset of patches by solving (7) with fixed $\mathbf{x}^{(1)}$ and $\mathbf{x}^{(2)}$ to obtain the coupled dictionaries. In Stage 2, we perform coupled sparse denoising with fixed dictionaries, where we fine tune the sparse representations $\mathbf{z}_{i j}, \mathbf{u}_{i j}$ and $\mathbf{v}_{i j}$ for each patch in order to remove aliasing and noise. Stage 3 enforces data consistency between the denoised image and the measurements in the $k$ space domain. Next, we describe each of these stages.

Stage 1) Coupled Dictionary Learning. In the first outer iteration, $\mathbf{x}^{(1)}$ and $\mathbf{x}^{(2)}$ are initialized as $\mathbf{F}_{u 1}^{H} \mathbf{y}^{(1)}$ and $\mathbf{F}_{u 2}^{H} \mathbf{y}^{(2)}$, respectively, where $\mathbf{F}_{u 1}^{H}$ (or $\mathbf{F}_{u 2}^{H}$ ) represents interpolating $\mathbf{y}^{(1)}$ (or $\mathbf{y}^{(2)}$ ) with zeros on non-sampled positions and then performing inverse Fourier transform. That is, $\mathbf{x}^{(1)}$ and $\mathbf{x}^{(2)}$ are set to be equal to the inverse DFT of their zero-filled Fourier measurements for Cartesian sampling, and are set to be the inverse NUFFT [39] of their $k$-space samples for sampling on a non-Cartesian grid. In the remaining outer iterations, $\mathbf{x}^{(1)}$ and $\mathbf{x}^{(2)}$ will be the output of Stage 3 ) from the previous outer iteration. For fixed $\mathbf{x}^{(1)}$ and $\mathbf{x}^{(2)}$, we attempt to solve (7) with respect to $\boldsymbol{\alpha}=\left(\mathbf{z}_{i j}, \mathbf{u}_{i j}, \mathbf{v}_{i j}\right)$ and $\mathbf{D}=\left(\boldsymbol{\Phi}_{c}, \boldsymbol{\Phi}, \boldsymbol{\Psi}_{c}, \boldsymbol{\Psi}\right)$ via alternating minimization. First, we solve the sparse coding problem (8) to update sparse representations for fixed dictionaries, and next we solve problem (9) to update dictionaries for fixed sparse representations.

A. Sparse Coding:

$$
\begin{aligned}
& \underset{\mathbf{z}_{i j}, \mathbf{u}_{i j}, \mathbf{v}_{i j}}{\operatorname{minimize}} \sum_{i j}\left\{\left\|\mathbf{R}_{i j} \mathbf{x}^{(1)}-\left(\boldsymbol{\Psi}_{c} \mathbf{z}_{i j}+\boldsymbol{\Psi} \mathbf{u}_{i j}\right)\right\|_{2}^{2}\right. \\
&\left.+\left\|\mathbf{R}_{i j} \mathbf{x}^{(2)}-\left(\boldsymbol{\Phi}_{c} \mathbf{z}_{i j}+\boldsymbol{\Phi} \mathbf{v}_{i j}\right)\right\|_{2}^{2}\right\} \\
& \text { subject to }\left\|\mathbf{z}_{i j}\right\|_{0} \leq s_{c},\left\|\mathbf{u}_{i j}\right\|_{0} \leq s_{1},\left\|\mathbf{v}_{i j}\right\|_{0} \leq s_{2}, \forall i, j .
\end{aligned}
$$

The sparse coding step is solved using the orthogonal matching pursuit (OMP) [37], [40]. 
B. Dictionary Update:

$$
\begin{aligned}
\underset{\boldsymbol{\Psi}_{c}, \boldsymbol{\Psi}, \mathbf{\Phi}_{c}, \mathbf{\Phi}}{\operatorname{minize}} \sum_{i j}\left\{\left\|\mathbf{R}_{i j} \mathbf{x}^{(1)}-\left(\boldsymbol{\Psi}_{c} \mathbf{z}_{i j}+\mathbf{\Psi} \mathbf{u}_{i j}\right)\right\|_{2}^{2}\right. \\
\left.+\left\|\mathbf{R}_{i j} \mathbf{x}^{(2)}-\left(\boldsymbol{\Phi}_{c} \mathbf{z}_{i j}+\mathbf{\Phi} \mathbf{V}_{i j}\right)\right\|_{2}^{2}\right\} \\
\text { subject to }\left\|\left[\begin{array}{l}
\boldsymbol{\psi}_{c k} \\
\boldsymbol{\phi}_{c k}
\end{array}\right]\right\|_{2}^{2} \leq 1,\left\|\boldsymbol{\psi}_{k}\right\|_{2}^{2} \leq 1,\left\|\boldsymbol{\phi}_{k}\right\|_{2}^{2} \leq 1, \forall k .
\end{aligned}
$$

The dictionary update is adapted from the Block Coordinate Descent [37], [41], where the dictionaries are updated one pair of atoms at a time ${ }^{2}$. The key idea of our coupled dictionary learning is to train the coupled dictionaries simultaneously and train the distinct dictionaries individually. Note that, since a single image contains a large number of patches, to save time in stage 1), we randomly select only a subset of the patches as a training dataset $\mathbf{X}^{(1)}=\left[\cdots, \mathbf{x}_{i j}^{(1)}, \cdots\right]$ and $\mathbf{X}^{(2)}=\left[\cdots, \mathbf{x}_{i j}^{(2)}, \cdots\right]$ which are updated in each outer iteration. The learning algorithm is shown in Algorithm 2. More details are provided in Appendix A.

Stage 2) Coupled Sparse Denoising. As the sparse representations computed in Stage 1) are associated only with a subset of the collection of image patches, we need to update the sparse representations of the remaining patches. In addition, we also introduce linearly decreasing error thresholds to fine tune the sparse representations, since our experiments have shown that this improves the de-aliasing and denoising performance. In particular, we give priority to updating the common sparse representations associated with the various image patches by performing the following optimization:

$$
\begin{aligned}
& \min _{\mathbf{z}_{i j}} \max \left\{\left\|\mathbf{R}_{i j} \mathbf{x}^{(1)}-\mathbf{\Psi}_{c} \mathbf{z}_{i j}\right\|_{2}^{2}+\left\|\mathbf{R}_{i j} \mathbf{x}^{(2)}-\mathbf{\Phi}_{c} \mathbf{z}_{i j}\right\|_{2}^{2}, \epsilon_{c}\right\} \\
& \text { s.t. }\left\|\mathbf{z}_{i j}\right\|_{0} \leq s_{c} .
\end{aligned}
$$

We then update the distinct sparse representations associated with the various target image patches by solving:

$$
\begin{array}{ll}
\min _{\mathbf{u}_{i j}} & \max \left\{\left\|\mathbf{R}_{i j} \mathbf{x}^{(1)}-\mathbf{\Psi}_{c} \mathbf{z}_{i j}-\mathbf{\Psi} \mathbf{u}_{i j}\right\|_{2}^{2}, \epsilon_{1}\right\} \\
\text { s.t. } & \left\|\mathbf{u}_{i j}\right\|_{0} \leq s_{1},
\end{array}
$$

and

$$
\begin{array}{ll}
\min _{\mathbf{v}_{i j}} & \max \left\{\left\|\mathbf{R}_{i j} \mathbf{x}^{(2)}-\mathbf{\Phi}_{c} \mathbf{z}_{i j}-\mathbf{\Phi} \mathbf{v}_{i j}\right\|_{2}^{2}, \epsilon_{2}\right\} \\
\text { s.t. } & \left\|\mathbf{v}_{i j}\right\|_{0} \leq s_{2} .
\end{array}
$$

Here $\epsilon_{c}, \epsilon_{1}$ and $\epsilon_{2}$ denote the error thresholds which are used together with $s_{c}, s_{1}$ and $s_{2}$ in OMP as the stopping criteria.

The above formulations imply that once the objective value for the $(i, j)$-th patch decreases below the expected error threshold, OMP stops early. In addition, as the quality of the reconstructed target image improves as the outer iterations proceed, we decrease the thresholds $\epsilon_{c}, \epsilon_{1}$ and $\epsilon_{2}$ linearly at each outer iteration. In practice, this strategy accelerates the algorithm, and also allows to dynamically control the sparsity of each patch more effectively.

Given the sparse representations $\mathbf{z}_{i j}, \mathbf{u}_{i j}$ and $\mathbf{v}_{i j}$, we obtain each denoised patch as $\hat{\mathbf{x}}_{i j}^{(1)}=\boldsymbol{\Psi}_{c} \mathbf{z}_{i j}+\boldsymbol{\Psi} \mathbf{u}_{i j}$ and $\hat{\mathbf{x}}_{i j}^{(2)}=$ $\boldsymbol{\Phi}_{c} \mathbf{z}_{i j}+\boldsymbol{\Phi} \mathbf{v}_{i j}$, where $\boldsymbol{\Psi}_{c}, \boldsymbol{\Psi}, \boldsymbol{\Phi}_{c}$ and $\boldsymbol{\Phi}$ are dictionaries learned in Stage 1).

\footnotetext{
${ }^{2}$ Compared to updating full dictionaries at once using least square, these sequential updates reduce the computational complexity from $O\left(K^{2} N\right)$ to $O(n K N)$, as well as reduce the memory requirements. However, they cannot be parallelized.
}

Stage 3) Enforcing $k$-space Consistency. Finally, in this stage, we enforce consistency between the denoised image and its measurements in the $k$-space domain. Taking contrast $\mathbf{x}^{(1)}$ as an example, we solve a least squares problem:

$$
\min _{\mathbf{x}^{(1)}} \sum_{i j}\left\|\mathbf{R}_{i j} \mathbf{x}^{(1)}-\hat{\mathbf{x}}_{i j}^{(1)}\right\|_{2}^{2}+\nu_{1}\left\|\mathbf{F}_{u 1} \mathbf{x}^{(1)}-\mathbf{y}^{(1)}\right\|_{2}^{2},
$$

where, $\hat{\mathbf{x}}_{i j}^{(1)}$ is a denoised patch obtained from Stage 2.

As in [2], we assume that patches wrap around at image boundaries (which implies that the number of overlapping patches occurring at each pixel is equal). Then, for sampling schemes on a uniform grid of the $k$-space, such as Gaussian random 2D sampling and Cartesian 1D sampling, we obtain the solution as follows:

$$
\tilde{\mathbf{x}}^{(1)}=\mathbf{F}^{H} \tilde{\mathbf{y}}^{(1)},
$$

where $\mathbf{F}^{H}$ denotes the conjugate of the Fourier transform matrix $\mathbf{F}$, and $\tilde{\mathbf{y}}^{(1)} \in \mathcal{C}^{N}$ denotes the updated $k$-space, where the elements are given by:

$$
\tilde{\mathbf{y}}_{[p]}^{(1)}=\left\{\begin{array}{ll}
\left(\mathbf{F} \hat{\mathbf{x}}^{(1)}\right)_{[p]}, & {[p] \notin \Omega^{(1)}} \\
\frac{1}{1+\tilde{\nu}_{1}}\left(\mathbf{F} \hat{\mathbf{x}}^{(1)}+\tilde{\nu}_{1} \mathbf{F} \mathbf{F}_{u 1}^{H} \mathbf{y}^{(1)}\right)_{[p]}, & {[p] \in \Omega^{(1)}}
\end{array} .\right.
$$

Here, $\tilde{\nu}_{1}=\nu_{1} / \beta, \hat{\mathbf{x}}^{(1)}=\frac{1}{\beta} \sum_{i j} \mathbf{R}_{i j}^{H} \hat{\mathbf{x}}_{i j}^{(1)} \in \mathcal{C}^{N}$ is the denoised image, $\beta$ is the number of overlapping patches at the corresponding pixel location in $\mathbf{x}^{(1)}, \Omega^{(1)} \in \mathcal{R}^{m_{1}}$ denotes the subset of $k$-space that has been sampled, and $[p]$ denotes the location in the vectorized $k$-space. Specifically, if location $[p]$ is not sampled, i.e. $[p] \notin \Omega^{(1)}$, we use the Fourier transform results of the denoised image $\hat{\mathbf{x}}^{(1)}$ to interpolate this location. Otherwise, if location $[p]$ is sampled, i.e. $[p] \in \Omega^{(1)}$, we consider both the Fourier transform results of the denoised image $\hat{\mathbf{x}}^{(1)}$ and original samples, and use their weighted sum to interpolate this location. Equation (15) is a kind of reformulation of Equation (9) in [2], and more details on the derivation and analysis can be found in Appendix A, as well as referring [2].

For sampling schemes on a non-uniform grid of the $k$ space, such as radial sampling, we propose to perform data consistency via a gradient step. Specifically, we compute the difference between the forward NUFFT of the denoised image $\hat{\mathbf{x}}^{(1)}$ and the groundtruth $k$-space samples $\mathbf{y}^{(1)}$. Then, the difference in $k$-space is transformed to the image domain via inverse NUFFT to update the estimation by a gradient descent operation with step size $\eta$, as

$$
\tilde{\mathbf{x}}^{(1)}=\hat{\mathbf{x}}^{(1)}-\eta \mathbf{F}_{u 1}^{H}\left(\mathbf{F}_{u 1} \hat{\mathbf{x}}^{(1)}-\mathbf{y}^{(1)}\right) .
$$

For joint reconstruction, we repeat the above procedures to estimate $\mathbf{x}^{(2)}$. The overall process consisting of the three stages is repeated over a number of outer iterations, as summarized in Algorithm 1.

\section{Algorithm Complexity and Convergence Behaviour}

The computing cost of dictionary learning is mainly dominated by sparse coding using OMP. Therefore, the complexity of the proposed CDLMRI algorithm is $O\left(\delta n K\left(2 s_{c}+\right.\right.$ $\left.\left.s_{1}+s_{2}\right) L N T\right)$, where $O\left(2 \delta n K s_{c} L N T\right), O\left(\delta n K s_{1} L N T\right)$ and 
Input: A subset of estimated image patch pairs: $\mathbf{X}^{(1)}=$ $\left\{\mathbf{x}_{i j}^{(1)}\right\}, \mathbf{X}^{(2)}=\left\{\mathbf{x}_{i j}^{(2)}\right\} ;$ Sparse constraints: $s_{c}, s_{1}, s_{2} ;$ Number of iterations: $L$; Number of dictionary atoms: $K$.

Output: Coupled dictionaries: $\boldsymbol{\Psi}_{c}, \boldsymbol{\Phi}_{c}, \boldsymbol{\Psi}, \boldsymbol{\Phi}$.

Initialization: Initialize each dictionary with randomly selected $K$ patches of the corresponding contrast. Initialize all sparse representations with zeros.

\section{Optimization:}

1: for $l=1, \cdots, L$ do

2: a) Sparse Coding step. Apply OMP to estimate $\mathbf{Z}=$ $\left[\cdots, \mathbf{z}_{i j}, \cdots\right]$ given data $\mathbf{X}^{(1)}, \mathbf{X}^{(2)}$ and dictionaries $\boldsymbol{\Psi}_{c}, \boldsymbol{\Phi}_{c}$.

3: $\quad$ Initialize the active set $\Gamma=\emptyset$ and $\mathbf{z}_{i j} \leftarrow 0$.

4: $\quad$ while $|\Gamma|<s_{c}$ do

5: $\quad$ select a new coordinate $\hat{k}$ that leads to the smallest residual and then update the active set and the solution $\mathbf{z}_{i j}$ :

$$
\begin{aligned}
& (\hat{k}, \hat{\boldsymbol{\alpha}}) \in \underset{k \in \Gamma^{c}, \boldsymbol{\alpha} \in \mathcal{C}^{|\Gamma|+1}}{\arg \min }\left\|\left[\begin{array}{l}
\mathbf{x}_{i j}^{(1)} \\
\mathbf{x}_{i j}^{(2)}
\end{array}\right]-\left[\begin{array}{l}
\mathbf{\Psi}_{c} \\
\boldsymbol{\Phi}_{c}
\end{array}\right]_{\Gamma \cup\{k\}} \boldsymbol{\alpha}\right\|_{2}^{2} ; \\
& \Gamma \leftarrow \Gamma \cup\{\hat{k}\} ; \quad \mathbf{z}_{i j_{\Gamma}} \leftarrow \hat{\boldsymbol{\alpha}} ; \quad \mathbf{z}_{i j_{\Gamma^{c}}} \leftarrow 0 ;
\end{aligned}
$$

6: end while

7: $\quad$ Steps $3-6$ are repeated to estimate $\mathbf{U}=\left[\cdots, \mathbf{u}_{i j}, \cdots\right]$ and $\mathbf{V}=\left[\cdots, \mathbf{v}_{i j}, \cdots\right]$ given $\mathbf{X}^{(1)}-\boldsymbol{\Psi}_{c} \mathbf{Z}=\boldsymbol{\Psi} \mathbf{U}$ and
$\mathbf{X}^{(2)}-\boldsymbol{\Phi}_{c} \mathbf{Z}=\boldsymbol{\Phi} \mathbf{V}$, with sparsity constraints $s_{1}$ and $s_{2}$, respectively.

8: b) Dictionary Update step. This step updates the dictionaries for fixed sparse codes.

for $k=1, \cdots, K$ do

Update the $k$-th column of $\boldsymbol{\Psi}_{c}$ and $\boldsymbol{\Phi}_{c}$ as follows:

$$
\begin{aligned}
& \mathbf{d}_{k} \leftarrow\left(\left[\begin{array}{l}
\mathbf{X}^{(1)}-\mathbf{\Psi} \mathbf{U} \\
\mathbf{X}^{(2)}-\mathbf{\Phi} \mathbf{V}
\end{array}\right]-\left[\begin{array}{l}
\boldsymbol{\Psi}_{c} \\
\boldsymbol{\Phi}_{c}
\end{array}\right] \mathbf{Z}+\left[\begin{array}{l}
\boldsymbol{\psi}_{c k} \\
\boldsymbol{\phi}_{c k}
\end{array}\right] \mathbf{z}^{k}\right) \mathbf{z}^{k^{H}} \\
& {\left[\begin{array}{l}
\boldsymbol{\psi}_{c k} \\
\boldsymbol{\phi}_{c k}
\end{array}\right] \leftarrow \frac{\mathbf{d}_{k}}{\max \left(\left\|\mathbf{d}_{k}\right\|_{2}, \mathbf{z}^{\left.k^{k} \mathbf{z}^{k^{H}}\right)}\right.}}
\end{aligned}
$$

where $\mathbf{z}^{k}$ denotes the $k$-th row of $\mathbf{Z}$.

11: end for

12: $\quad$ for $k=1, \cdots, K$ do

13: Update the $k$-th column of $\boldsymbol{\Psi}$ and $\boldsymbol{\Phi}$ as follows:

$$
\begin{aligned}
& \boldsymbol{\psi}_{k} \leftarrow\left(\mathbf{X}^{(1)}-\boldsymbol{\Psi}_{c} \mathbf{Z}-\mathbf{\Psi} \mathbf{U}+\boldsymbol{\psi}_{k} \mathbf{u}^{k}\right) \mathbf{u}^{k^{H}} \\
& \boldsymbol{\phi}_{k} \leftarrow\left(\mathbf{X}^{(2)}-\mathbf{\Phi}_{c} \mathbf{Z}-\mathbf{\Phi} \mathbf{V}+\boldsymbol{\phi}_{k} \mathbf{v}^{k}\right) \mathbf{v}^{k^{H}} \\
& \boldsymbol{\phi}_{k} \leftarrow \frac{\boldsymbol{\phi}_{k}}{\max \left(\left\|\phi_{k}\right\|_{2}, \mathbf{u}^{k} \mathbf{u}^{k^{H}}\right)} ; \boldsymbol{\psi}_{k} \leftarrow \frac{\boldsymbol{\psi}_{k}}{\max \left(\left\|\boldsymbol{\psi}_{k}\right\|_{2}, \mathbf{v}^{k} \mathbf{v}^{k^{H}}\right)}
\end{aligned}
$$

where $\mathbf{u}^{k}$ and $\mathbf{v}^{k}$ denote the $k$-th row of $\mathbf{U}$ and $\mathbf{V}$, respectively.

\section{4: end for}

\section{5: end for}

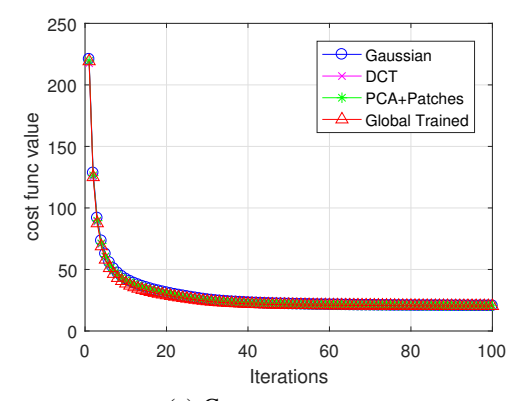

(a) Convergence curve

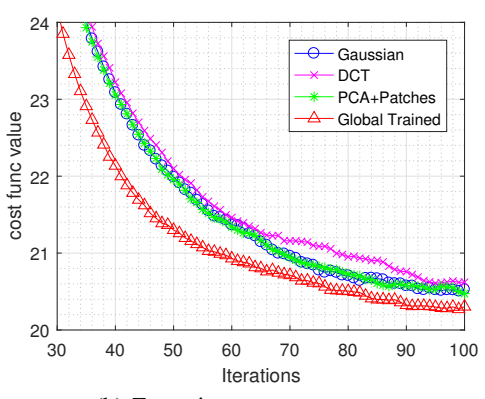

(b) Zoom-in convergence curve

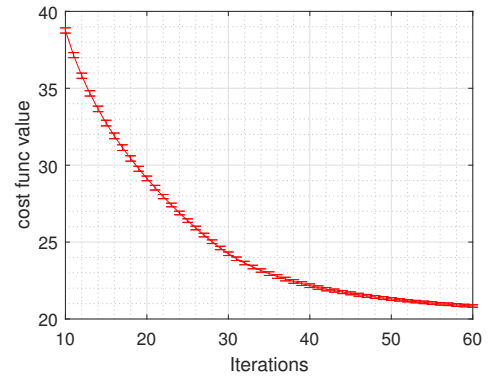

(c) Average convergence and s.d.

Figure 3: Convergence behaviour with respect to different dictionary initialization methods. (a) The cost function decreases monotonically along with the outer iterations. Such convergence behaviour applies to different dictionary initialization methods. (b) Zoom-in convergence curve for last few iterations. (c) The mean value and standard deviation (s.d.) for multiple repetitions.

$O\left(\delta n K s_{2} L N T\right)$ are the complexities of performing sparse coding with coupled dictionary pairs and two distinct dictionaries, respectively, with $L$ the number of training iterations, $\delta N$ the fraction $\delta$ of all $N$ patches used for dictionary learning, and $T$ the number of entire outer iterations. Thus, our complexity is on the same order as that of DLMRI, $O(\delta n K s L N T)$.

The proposed algorithm alternates between coupled dictionary learning, coupled sparse coding and enforcing k-space consistency. Assume that each step is performed successfully to decrease the total representation error, alternatively executing a series of such stages leads to a monotonic decrease in the nonnegative cost function. Furthermore, if one uses entire patches and provides a sufficient number of inner iterations for dictionary update $\left(\boldsymbol{\Psi}_{c}, \boldsymbol{\Psi}, \boldsymbol{\Phi}_{c}, \boldsymbol{\Phi}\right)$, sparse representation update $\left(\mathbf{z}_{i j}, \mathbf{u}_{i j}, \mathbf{v}_{i j}\right)$, and image update $\left(\mathbf{x}^{(1)}, \mathbf{x}^{(2)}\right)$ to guarantee sufficient decrease in costs, alternating optimization usually leads to convergence to local minima. Note that, the above claim depends on the success of coupled sparse coding which is more likely to be satisfied in circumstances of small sparsity. Otherwise, the convergence of coupled dictionary learning to a local minimum is not always guaranteed. When there is measurement noise during k-space sampling, the proposed method requires proper parameter tuning to balance between the denoised image and original k-space samples during the stage of enforcing k-space consistency, in order to guarantee 

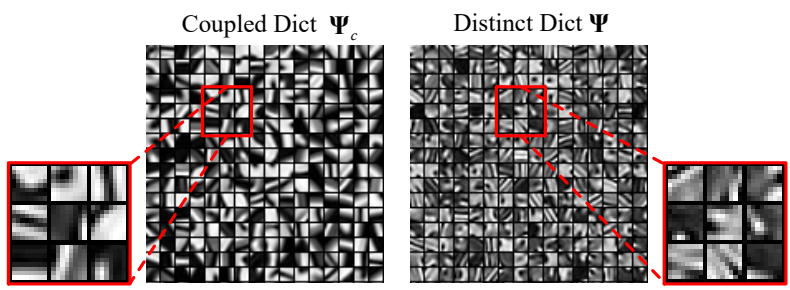

Coupled Dict $\boldsymbol{\Phi}$
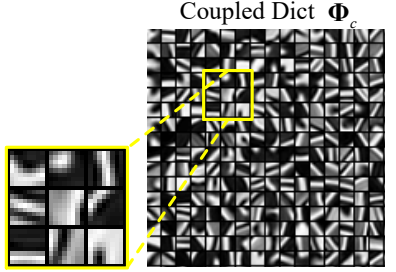

Distinct Dict $\boldsymbol{\Phi}$
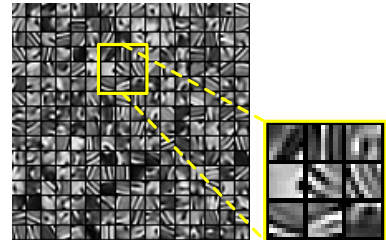

Figure 4: Learned coupled dictionaries from T1- and T2-weighted MRI contrasts; 256 atoms are shown. The top row displays the coupled and distinct dictionaries for the T1-weighted contrast. The bottom row displays dictionaries learned from corresponding T2weighted contrast. It can be seen that the atom pairs from coupled dictionaries $\boldsymbol{\Psi}_{c}, \boldsymbol{\Phi}_{c}$ capture associated edges, blobs and textures with the same direction and location, while $\boldsymbol{\Psi}, \boldsymbol{\Phi}$ represent the disparities.

that the updated estimation is always improved. More rigorous convergence analysis for such alternating methods remains to be investigated further.

Although theoretical convergence is not guaranteed, the convergence behaviour has been validated empirically on extensive experiments. In particular, we carried out a set of experiments to investigate the convergence behaviour of the proposed CDLMRI algorithm with respect to different dictionary initialization methods, such as Gaussian matrix, DCT matrix, PCA of image patches, and globally trained dictionaries. As shown in Fig. 3 (a) and (b), it is observed that various dictionary initialization methods can provide convergence. Using globally trained dictionaries which are learned on a set of synthetic MRI images from BrainWeb give slightly better convergence than the other 3 initialization methods. We also conducted multiple repetitions (200 times) of the experiments and computed the mean value and the standard deviation (s.d.) of RMSE, depicted by the curve and the error bar in Fig. 3 (c). It shows that the relative error (i.e. ratio of the mean value over the s.d.) is as small as $0.39 \%$.

\section{EXPERIMENTS}

We carried out a series of experiments to evaluate the performance of the proposed algorithm on guided and joint reconstruction. We examined a variety of MRI contrasts, under-sampling patterns and factors, with and without noise. Sampling schemes used in the experiments include 2D random sampling [10], Cartesian sampling with random phase encodes (1D random), and radial sampling with golden angle. The radial sampling with golden angle is implemented using the NUFFT package of Fessler et al. [39]. Similar to previous approaches [2], [8]-[10], [19], [21], complex-valued k-space data was acquired by retrospectively under-sampling the $2 \mathrm{D}$ discrete Fourier transform of magnitude MRIs. The MRI dataset includes both realistically simulated MR scans from BrainWeb $^{3}$ and real clinical MR scans used in [19], [21].

\footnotetext{
${ }^{3}$ http://brainweb.bic.mni.mcgill.ca/brainweb/
}
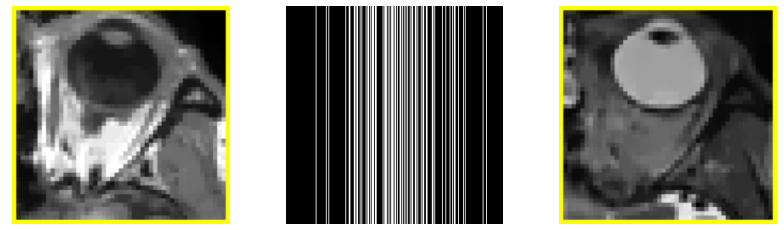

(a) Groundtruth T1 (left), Mask (middle), Guidance T2 (right)

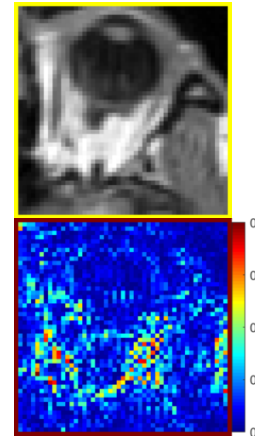

DLMRI

PSNR $=33.4 \mathrm{~dB}$

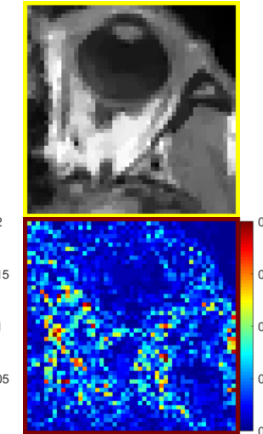

STVMRI

PSNR $=33.7 \mathrm{~dB}$

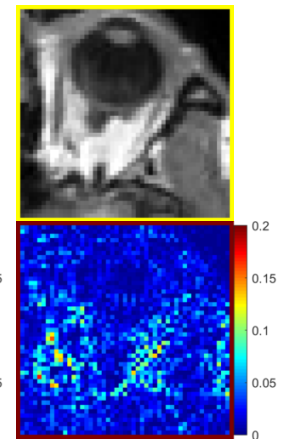

CDLMRI

PSNR = 36.1 dB (b) Reconstruction and error map of 3 algorithms

Figure 5: Guided reconstruction for T1, with fully-sampled T2 as reference with 4 fold Cartesian 1D random under-sampling. (a) groundtruth T1-weighted contrast, sampling mask and guidance T2weighted contrast. (b) reconstructed images and the corresponding residual error for single-contrast MRI reconstruction approach DLMRI [2], guided approach STVMRI [21], and the proposed CDLMRI. It can be seen that the proposed approach reliably reconstructs fine details and substantially suppresses aliasing, noise and artifacts, leading to the smallest residual error.

We compared our method against several representative algorithms, including DLMRI [2], STVMRI [21], RefMRI [19], and FJGP [24]. The implementation codes of these algorithms are available at the authors' websites. We use the built-in parameter settings in those implementations, which were tuned for the dataset we test all the algorithms on. We quantitatively assess image quality using two commonly used evaluation metrics, the Root Mean Squared Error (RMSE) between the original image and the reconstructed image, and the Peak Signal to Noise Ratio (PSNR), defined as PSNR $=20 \log _{10} \frac{\text { PeakVal }}{\text { RMSE }}$ where PeakVal stands for the pixel peak value. The experiments were conducted using MATLAB R2016b in a computer equipped with an Intel double-core i7-6500U CPU at $2.59 \mathrm{GHz}$ with $12 \mathrm{~GB}$ of memory, and 64-bit Windows 10 operating system.

In the experiments, the nominal values of the various parameters were set as image size $\sqrt{N} \times \sqrt{N}=256 \times 256$, patch size $\sqrt{n} \times \sqrt{n}=8 \times 8$, number of atoms in a dictionary $K=512$, number of inner iterations $L=50$, number of outer iterations $T=50$, sparsity constraints $s_{c}=\operatorname{ceil}(0.16 n), s_{1}=$ $s_{2}=\operatorname{ceil}\left(0.2 s_{c}\right)$, error thresholds $\epsilon_{c}=0.1 \downarrow 0.005$ (meaning: $\epsilon_{c}$ is set to 0.1 in the beginning and linearly decreases to 0.005 along the outer iterations.), $\epsilon_{1}=\epsilon_{2}=0.9 \epsilon_{c}, \nu_{1}=\nu_{2} \rightarrow \infty$ (for noise-free situation) or $\nu_{1}=\lambda / \sigma_{1}, \nu_{2}=\lambda / \sigma_{2}$ (for the noise scenarios, where $\sigma_{1}$ and $\sigma_{2}$, assumed to be known, are the standard deviations of the measurement noise, and $\lambda=140$ ), and gradient step $\eta=1.2$.

We first present the coupled dictionary learning results in Section IV-A to show that our algorithm is capable of 
capturing structure similarities and disparities between different MRI contrasts. Then, we present the results for guided reconstruction in Section IV-B where one has access to a fully sampled contrast to aid the reconstruction of the target contrast. Next, we repeat the experiments in Section IV-C for joint reconstruction where different MRI contrasts are undersampled and then reconstructed simultaneously. Appendix $\mathrm{B}$ includes additional experiments and an analysis of the performance sensitivity with respect to key parameters, such as acceleration factors, dictionary size, noise levels, etc.

\section{A. Coupled Dictionary Learning}

T1-weighted and T2-weighted MRI scans are two types of commonly used multi-contrast data. In general, T1-weighted MRI images highlight fat tissue, such as subcutaneous fat (SC fat) and bone marrow, and suppress water-based tissue, such as Cerebrospinal fluid (CSF). In contrast, T2-weighted MRI images highlight both fat tissue and water-based tissue. Therefore, T1-weighted and T2-weighted MRIs exhibit general reverse brightness in fat tissue and similar brightness in water-based tissue.

Fig. 4 shows the learned coupled dictionaries from T1and T2- weighted MRI images. It shows that the atom pairs from coupled dictionaries $\boldsymbol{\Psi}_{c}, \boldsymbol{\Phi}_{c}$ capture associated edges, blobs and textures with the same direction and location. Most of them exhibit considerable resemblance to each other, but with opposite intensity. This phenomenon is consistent with MRI characteristics determined by magnetization properties of tissue, such as CSF being dark in T1-weighted contrast and bright in T2-weighted. This outcome indicates that the coupled dictionaries are able to capture the similarities between T1-weighted and T2-weighted contrasts. In comparison, the learned distinct dictionaries $\boldsymbol{\Psi}, \boldsymbol{\Phi}$ represent the disparities of these modalities, and such disparities can be observed in the highlighted atoms in the figure. The training results illustrate that the learned dictionaries are adaptive to the data modalities. Moreover, they are capable of capturing the complicated mapping between T1/T2-weighted multi-contrast MRIs, thus better characterizing the inherent dependency relationship than predefined bases. Such adaptive dictionaries can be employed to effectively reduce aliasing, noise and other artifacts, as demonstrated in the following subsections.

\section{B. Guided Reconstruction}

We first consider the scenario in which one contrast is undersampled to serve as the target contrast to be reconstructed, while another one is fully-sampled as the guidance contrast to aid reconstruction.

1) Guided Reconstruction for T1, with $T 2$ as reference: In this experiment, we reconstruct a T1-weighted MRI from its under-sampled $k$-space measurements, with the aid of a T2-weighted fully-sampled MRI. This replicates the scenario in [21]. We compare our CDLMRI with SVTMRI [21] which uses a structure-guided total variation to transfer structural information across the different contrasts. We also compare the proposed approach with DLMRI [2] to illustrate the benefits of integrating a reference contrast in the reconstruction process.
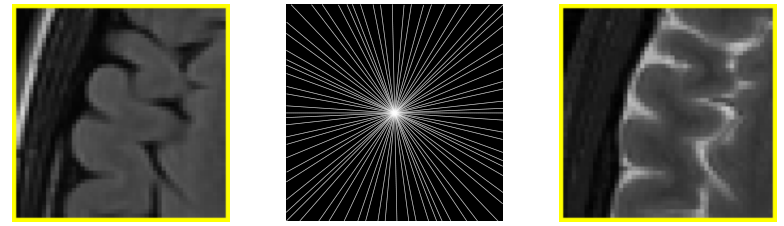

(a) Groundtruth FLAIR (left), Mask (middle), Guidance T2 (right)

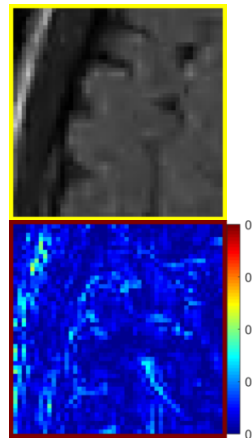

RefMRI

PSNR $=30.7 \mathrm{~dB}$

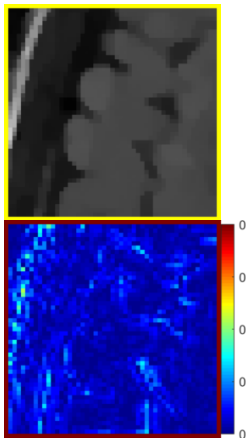

FJGP

PSNR $=32.1 \mathrm{~dB}$

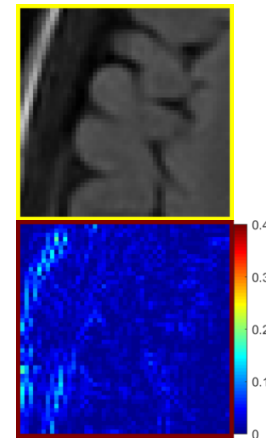

CDLMRI

PSNR = 34.6 dB (b) Reconstruction and error map of 3 algorithms

Figure 6: Guided reconstruction for FLAIR, with fullysampled T2 as reference with 6.67 fold radial under-sampling, using RefMRI [19], FJGP [24], and the proposed CDLMRI.

Fig. 5 shows reconstruction results for the scenario where a variable density Cartesian mask is employed for four fold under-sampling (i.e. $25 \% \quad k$-space data) on the target T1weighted contrast, with a fully sampled T2-weighted MRI for guidance contrast. It can be seen that the reconstructed target contrast and its corresponding residual from DLMRI [2] exhibit undesirable aliasing, noise and blurred areas. In comparison, the edges, outlines and textures in the reconstructed image from STVMRI [21] are sharper, thereby more visually appealing in high-frequency regions. However, it is also noticed that these high-frequency regions tend to be oversharpened, thus introducing noticeable artifacts. In contrast, the proposed CDMRI approach substantially attenuates aliasing and noise. At the same time, CDLMRI reliably restores fine details without introducing noticeable artifacts, leading to a more comprehensive and interpretable reconstruction. The performance improvement is also demonstrated by the PSNR values with $2.7 \mathrm{~dB}$ and $2.4 \mathrm{~dB}$ improvement over DLMRI [2] and STVMRI [21], respectively.

2) Guided Reconstruction for FLAIR, with T2 as reference: In this experiment, the task is to reconstruct a fluid-attenuated inversion recovery (FLAIR) contrast, from under-sampled measurements, by capitalizing on the similarity with a fullysampled T2-weighted contrast. The data was collected by the authors of [19] and made available on the author's website. We sampled $15 \%$ (i.e. 6.67 fold under-sampling) of the FLAIR $k$ space with radial sampling. As mentioned before, the radial sampling pattern with golden angle is implemented using NUFFT package of Fessler et al. [39], which is more practical than the radial sampling pattern used in STVMRI [21]. As STVMRI [21] and DLMRI [2] cannot apply practical radial sampling directly, the two methods are not included in our comparisons. We compare CDLMRI with RefMRI [19] and 


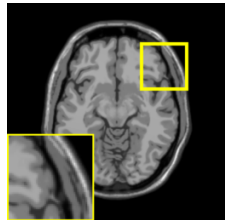

Groundtruth T1

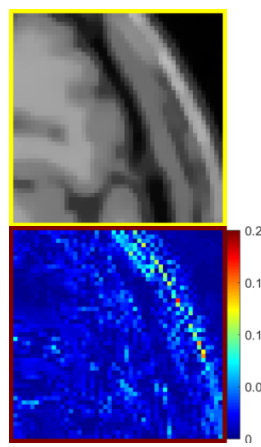

FJGP

PSNR $=36.1 \mathrm{~dB}$
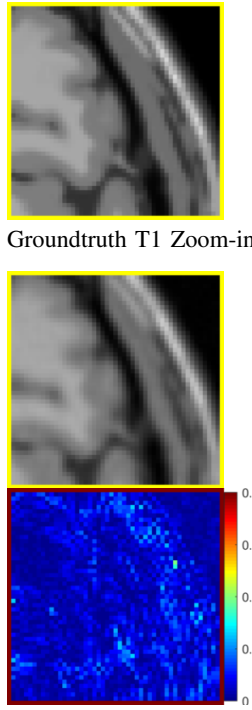

DLMRI

PSNR $=37.8 \mathrm{~dB}$
Groundtruth T1 Zoom-in

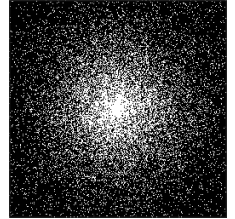

Sampling mask

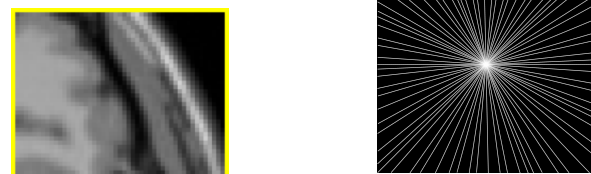

Groundtruth FLAIR Radial sampling mask

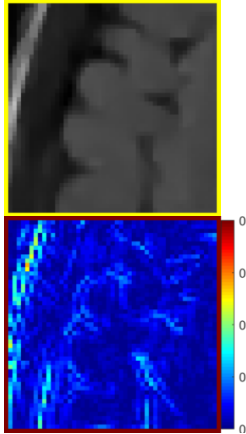

FJGP

PSNR $=31.3 \mathrm{~dB}$

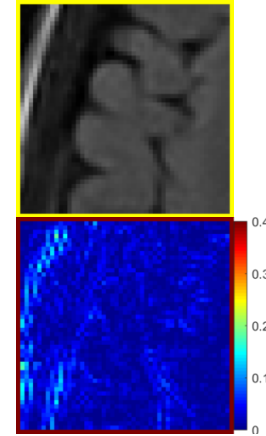

CDLMRI

PSNR $=\mathbf{3 4 . 2} \mathbf{d B}$ (a) reconstruction for FLAIR contrast

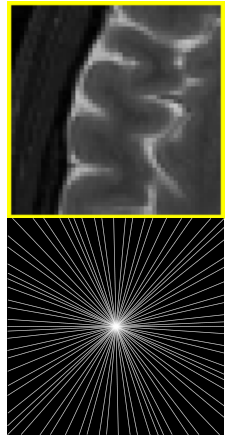

Groundtruth T2 / Radial sampling mask

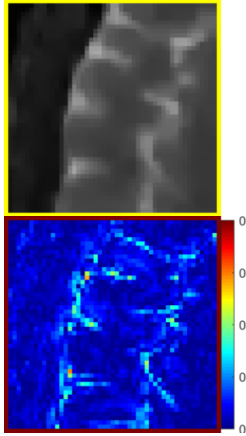

FJGP

PSNR $=31.1 \mathrm{~dB}$

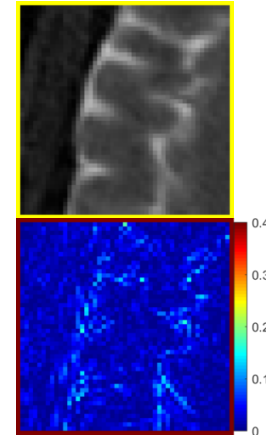

CDLMRI

$\mathrm{PSNR}=33.0 \mathrm{~dB}$ (b) reconstruction for $\mathrm{T} 2$-weighted contrast

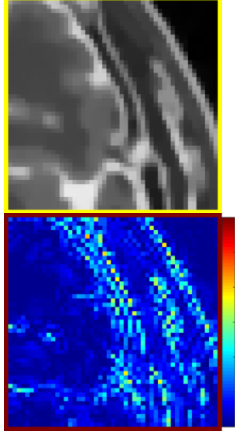

FJGP

PSNR $=30.8 \mathrm{~dB}$
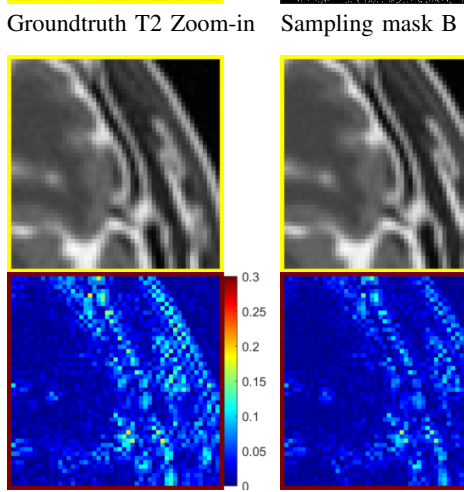

DLMRI

PSNR $=31.2 \mathrm{~dB}$

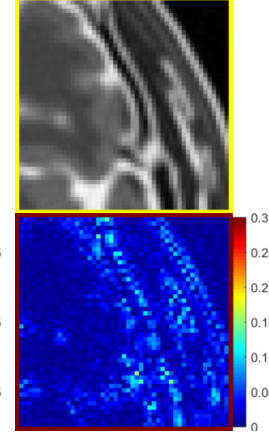

CDLMRI

PSNR $=33.4 \mathrm{~dB}$ (b) reconstruction for $\mathrm{T} 2$-weighted contrast

Figure 7: Joint reconstruction for both T1- and T2-weighted MRIs with 5 fold 2D random under-sampling using FJGP [24], DLMRI [2] and proposed CDLMRI.

FJGP [24] which use the same guidance contrast and sampling pattern as ours for reconstructing FLAIR.

Fig. 6 shows the groundtruth FLAIR contrast and fully sampled T2-weighted version, as well as the reconstructed images and corresponding residual error maps from RefMRI [19], FJGP [24] and CDLMRI. It can be clearly seen that FLAIR reconstruction using CDLMRI is clearer, sharper and more interpretable than the reconstruction by the two other methods. In particular, the gyrus and sulcus, shown in the zoom-in region, appear less obscured than those in RefMRI [19], FJGP [24], indicating the advantage of CDLMRI in restoring fine features and suppressing noise. The residual error maps, shown on the same scale, indicate that CDLMRI leads to the smallest reconstruction error. The performance improvement
Figure 8: Joint reconstruction for both FLAIR and T2weighted MRIs with 6.67 fold radial under-sampling using FJGP [24] and the proposed CDLMRI. (a) groundtruth and reconstruction for FLAIR contrast. (b) groundtruth and reconstruction for T2 weighted contrast.

is also demonstrated by the impressive PSNR gains with 3.9 $\mathrm{dB}$ and $2.5 \mathrm{~dB}$ improvement over RefMRI [19] and FJGP [24], respectively, for $15 \%$ (i.e. 6.67 fold) under-sampling. This advantage also holds for other under-sampling folds, such as 2 fold leading to PSNR gains of $4.4 \mathrm{~dB}$ and $4.2 \mathrm{~dB}$ better than RefMRI [19] and FJGP [24], respectively, as shown in Appendix B.

\section{Joint Reconstruction}

We now explore the scenario in which both contrasts are under-sampled in their $k$-space and we reconstruct them jointly using the proposed CDLMRI. We compare with FJGP [24] which also performs joint reconstruction in the current situation, and also with DLMRI [2] which performs separate reconstruction for both contrasts from their $k$-space samples. The experimental setting is similar to the one in Section IV-B.

1) Joint Reconstruction for T1- and T2-weighted: In this experiment, both T1- and T2-weighted contrasts are undersampled using two different 2D random sampling masks with the same sampling ratio. Then, we jointly reconstruct both contrasts from their $k$-space samples. The visual performance, shown in Fig. 7, demonstrates that the images reconstructed by DLMRI [2] and FJGP [24] exhibit noticeable blurred or over-smoothed regions, whereas the reconstructed contrasts by CDLMRI are sharper and visually more appealing. The 
performance improvement is also demonstrated by the PSNR gains.

2) Joint Reconstruction for FLAIR and T2-weighted: In this experiment, both FLAIR and T2-weighted contrasts are under-sampled using the same radial sampling, and are then jointly reconstructed. Fig. 8 shows the visual performance, where it can be seen that the proposed CDLMRI outperforms FJGP [24] in terms of both visual and quantitative metrics. The zoomed gyrus and sulcus regions in our reconstruction appear clearer and less obscure than in FJGP [24]. In addition, the residual error maps, shown on the same scale, also indicate that CDLMRI leads to the smallest reconstruction error.

To summarize, the advantage of CDLMRI is the ability of learning adaptive coupled dictionaries that are capable of effectively capturing salient features and critical correlations between the target and the guidance MRI contrasts in their sparse transform domains. By employing learned priors, CDLMRI effectively reduces streaking artifacts for Cartesian 1D random sampling, spiny artifacts for radial sampling, and incoherent aliasing for 2D random sampling compared to competing methods.

\section{Discussion}

We also compare CDLMRI with other state-of-the-art methods (please refer to Appendix B Section D and E for detailed results). Further, we show in Appendix B Section F that the proposed method can also be applied to Magnetic Resonance Fingerprinting (MRF) and achieves decent performance in this quantitative MR imaging task that acquires tissue's quantitative parameters (e.g. longitudinal relaxation time [T1] and transverse relaxation time [T2]) simultaneously.

We point out that the computation time of our method is higher compared to CSMRI approaches. However, this can be improved with parallel computing for sparse coding operations. Our experiments were performed with retrospective under-sampling of inverse-Fourier transformed magnitude images, rather than under-sampling raw complex MRI data. While this does not reflect the real MRI acquisition scenario perfectly, retrospective under-sampling is standard practice adopted by all methods to allow a fair comparison between them.

The proposed data model may have other variants for taking advantage of the common information among multi-contrast images. One of them is based on an assumption that they share a common dictionary instead of common sparse representations. Our analysis shows that this reduces to a model similar to classic dictionary learning, and the contribution from the different modalities is loosely coupled during the dictionary learning. More detailed analysis can be found in Appendix C. Section A.

Our approach works with image patches during coupled dictionary learning and image reconstruction. A large image may result in a large number of patches and consume a considerable amount of memory. In order to alleviate the memory burden, we rely on a few tricks, such as randomly producing a fraction of patches and removing patches with small variance during coupled dictionary learning, producing a moderate number of patches every time for reconstruction. More detailed description can be found in Appendix C. Section C.

The proposed data model for CDLMRI admits a resemblance to that in CDLSR [38] to some extent, as they both exploit the assumption that different data modalities may share common components represented by identical sparse codes. However, we highlight that there exists a variety of differences between our CDLMRI approach and CDLSR not only in the data model, but also including the task, goal, training strategy, and optimization method. More detailed description can be found in Appendix C. Section D.

Since the data in the experiments is from rasterized images, there might exist inverse crime [42] which may lead to artificially good results due to the neglect of the aliasing that is inherent to spatial discretization. This issue influences both our approach and competing approaches. More discussion can be found in Appendix C. Section E.

We used the same setting as in other literature and did not consider the possible effect of nonlinear effects. To reduce the distortion due to the nonlinear effects in practice, one may apply some strategies [43]-[46], for example, using higher performance hardware, parallel imaging, etc. (See Appendix C. Section F for details).

We also discussed possibilities and limitations of extending our method to multi-coil multi-contrast MR imaging and phase image reconstruction in Appendix $\mathrm{C}$ Section $\mathrm{G}$ and $\mathrm{H}$.

\section{CONCLUSION}

We proposed a coupled dictionary learning based multicontrast MRI reconstruction framework that capitalizes on not only patch-based sparsity, but also structure similarity across different MRI contrasts. The proposed framework enables the learned adaptive coupled dictionaries to capture both the similarities and disparities between different contrasts, and exploits such beneficial dependency information to improve reconstruction performance. Practical experiments on a variety of MRI contrasts, sampling schemes and under-sampling factors demonstrate the superior performance of our design with respect to competing methods. In future work, we may consider extending the proposed framework to other multi-modal image restoration, such as joint positron emission tomography (PET) and computed tomography (CT) reconstruction, as well as parallel multi-contrast MR imaging.

\section{REFERENCES}

[1] M. Lustig, D. Donoho, and J. Pauly, "Sparse MRI: The application of compressed sensing to rapid MR imaging," Magn. Reson. Med., vol. 58, pp. 1182-1195, 2007.

[2] S. Ravishankar and Y. Bresler, "MR image reconstruction from highly undersampled k-space data by dictionary learning," IEEE Trans. Med. Imag., vol. 30, no. 5, pp. 1028-1041, 2011.

[3] J. Caballero, A. N. Price, D. Rueckert, and J. V. Hajnal, "Dictionary learning and time sparsity for dynamic MR data reconstruction," IEEE Trans. Med. Imag., vol. 33, no. 4, pp. 979-994, 2014.

[4] D. Donoho, "Compressed sensing," IEEE Trans. Info. Theory, vol. 52, no. 4, pp. 1289-1306, 2006.

[5] E. Candès, J. Romberg, and T. Tao, "Robust uncertainty principles: Exact signal reconstruction from highly incomplete frequency information," IEEE Trans. Info. Theory, vol. 52, no. 2, pp. 489-509, 2006. 
[6] Y. C. Eldar and G. Kutyniok, Compressed sensing: theory and applications. Cambridge University Press, 2012.

[7] Y. C. Eldar, Sampling Theory: Beyond Bandlimited Systems. Cambridge University Press, 2015.

[8] S. Ma, W. Yin, Y. Zhang, and A. Chakraborty, "An efficient algorithm for compressed MR imaging using total variation and wavelets," in Proc. IEEE Conf. Comput. Vision Pattern Recog. IEEE, 2008, pp. 1-8.

[9] J. Yang, Y. Zhang, and W. Yin, "A fast alternating direction method for tv11-12 signal reconstruction from partial fourier data," IEEE Journal of Selected Topics in Signal Processing, vol. 4, no. 2, pp. 288-297, 2010.

[10] J. Trzasko and A. Manduca, "Highly undersampled magnetic resonance image reconstruction via homotopic $\ell_{0}$-minimization," IEEE Trans. Med. Imag., vol. 28, no. 1, pp. 106-121, 2009.

[11] Y. Wang and L. Ying, "Compressed sensing dynamic cardiac cine MRI using learned spatiotemporal dictionary." IEEE Trans. Biomed. Engineering, vol. 61, no. 4, pp. 1109-1120, 2014.

[12] Y. Huang, J. Paisley, Q. Lin, X. Ding, X. Fu, and X.-P. Zhang, "Bayesian nonparametric dictionary learning for compressed sensing MRI," EEE Trans. Image Process., vol. 23, no. 12, pp. 5007-5019, 2014

[13] M. Aharon, M. Elad, and A. Bruckstein, "K-svd: An algorithm for designing overcomplete dictionaries for sparse representation," IEEE Trans. Signal Process., vol. 54, no. 11, pp. 4311-4322, 2006.

[14] J. Sun, H. Li, Z. Xu et al., "Deep ADMM-Net for compressive sensing MRI," in Advances in Neural Information Processing Systems, 2016, pp. $10-18$.

[15] C. M. Hyun, H. P. Kim, S. M. Lee, S. Lee, and J. K. Seo, "Deep learning for undersampled MRI reconstruction," arXiv preprint arXiv:1709.02576, 2017

[16] J. Schlemper, J. Caballero, J. V. Hajnal, A. Price, and D. Rueckert, "A deep cascade of convolutional neural networks for MR image reconstruction," in International Conference on Information Processing in Medical Imaging. Springer, 2017, pp. 647-658.

[17] G. Yang, S. Yu et al., "Dagan: Deep de-aliasing generative adversarial networks for fast compressed sensing MRI reconstruction," IEEE Trans. Med. Imaging, 2017.

[18] L. Sun, Z. Fan, Y. Huang, X. Ding, and J. Paisley, "Compressed sensing MRI using a recursive dilated network," in AAAI, 2018.

[19] L. Weizman, Y. C. Eldar, and D. Ben Bashat, "Reference-based MRI," Medical physics, vol. 43, no. 10, pp. 5357-5369, 2016.

[20] L. Weizman, Y. C. Eldar, and D. Ben Bashat, "Compressed sensing for longitudinal MRI: An adaptive-weighted approach," Medical physics, vol. 42, no. 9, pp. 5195-5208, 2015.

[21] M. J. Ehrhardt and M. M. Betcke, "Multicontrast MRI reconstruction with structure-guided total variation," SIAM J. Imaging Sci., vol. 9, no. 3, pp. 1084-1106, 2016.

[22] B. Bilgic, V. K. Goyal, and E. Adalsteinsson, "Multi-contrast reconstruction with bayesian compressed sensing," Magn. Reson. Med., vol. 66, no. 6, pp. 1601-1615, 2011.

[23] X. Qu, Y. Hou, F. Lam, D. Guo, J. Zhong, and Z. Chen, "Magnetic resonance image reconstruction from undersampled measurements using a patch-based nonlocal operator," ed. Image Anal., vol. 18, no. 6, pp. 843-856, 2014.

[24] J. Huang, C. Chen, and L. Axel, "Fast multi-contrast MRI reconstruction," Magn. Reson. Imaging, vol. 32, no. 10, pp. 1344-1352, 2014.

[25] I. Chatnuntawech, A. Martin, B. Bilgic, K. Setsompop, E. Adalsteinsson, and E. Schiavi, "Vectorial total generalized variation for accelerated multi-channel multi-contrast MRI," Magn. Reson. Imaging, vol. 34, no. 8, pp. 1161-1170, 2016.

[26] X. Peng, H.-Q. Du, F. Lam, S. D. Babacan, and Z.-P. Liang, "Referencedriven MR image reconstruction with sparsity and support constraints," in Proc. IEEE Int. Symp. Biomed. Imaging. IEEE, 2011, pp. 89-92.

[27] H. Du and F. Lam, "Compressed sensing MR image reconstruction using a motion-compensated reference," Magn. Reson. Imaging, vol. 30, no. 7, pp. 954-963, 2012.

[28] Z. Lai, X. Zhang et al., "Joint sparse reconstruction of multi-contrast MRI images with graph based redundant wavelet transform," $B M C$ medical imaging, vol. 18, no. 1, p. 7, 2018.

[29] L. Sun, Z. Fan, Y. Huang, X. Ding, and J. Paisley, "A deep information sharing network for multi-contrast compressed sensing MRI reconstruction," arXiv preprint arXiv:1804.03596, 2018.

[30] A. G. Christodoulou, J. L. Shaw et al., "Magnetic resonance multitasking for motion-resolved quantitative cardiovascular imaging," Nat. Biomed. Eng., vol. 2, no. 4, p. 215, 2018.

[31] A. Bustin, G. Lima da Cruz, O. Jaubert, K. Lopez, R. M. Botnar, and C. Prieto, "High-dimensionality undersampled patch-based reconstruction (hd-prost) for accelerated multi-contrast MRI," Magn. Reson. Med. 2019.
[32] Z.-P. Liang and P. C. Lauterbur, Principles of magnetic resonance imaging: a signal processing perspective. SPIE Opti. Eng. Press, 2000.

[33] D. W. McRobbie, E. A. Moore, M. J. Graves, and M. R. Prince, MRI from Picture to Proton. Cambridge university press, 2007.

[34] X. Qu, D. Guo et al., "Undersampled MRI reconstruction with patchbased directional wavelets," Magn. Reson. Imaging.

[35] Y. Liu, Z. Zhan, J.-F. Cai, D. Guo, Z. Chen, and X. Qu, "Projected iterative soft-thresholding algorithm for tight frames in compressed sensing magnetic resonance imaging," IEEE Trans. Med. Imaging, vol. 35, no. 9, pp. 2130-2140, 2016.

[36] Z. Zhan, J.-F. Cai, D. Guo, Y. Liu, Z. Chen, and X. Qu, "Fast multiclass dictionaries learning with geometrical directions in MRI reconstruction," IEEE Trans. Biomed. Eng., vol. 63, no. 9, pp. 1850-1861, 2016.

[37] J. Mairal, F. Bach, J. Ponce et al., "Sparse modeling for image an vision processing," Found. Trends Comput. Graph. Vis., vol. 8, no. 2-3, pp. 85-283, 2014

[38] P. Song, J. F. Mota, N. Deligiannis, and M. R. Rodrigues, "Coupled dictionary learning for multimodal image super-resolution," in IEEE Global Conf. Signal Inform. Process. IEEE, 2016, pp. 162-166.

[39] J. A. Fessler and B. P. Sutton, "Nonuniform fast fourier transforms using min-max interpolation," IEEE Trans. Sig. Proc., vol. 51, no. 2, pp. 560 574,2003

[40] J. A. Tropp and A. C. Gilbert, "Signal recovery from random measurements via orthogonal matching pursuit," IEEE Trans. Inform. Theory, vol. 53, no. 12, pp. 4655-4666, 2007.

[41] J. Mairal, F. Bach, J. Ponce, and G. Sapiro, "Online learning for matrix factorization and sparse coding," J. Mach. Learn. Res., vol. 11, pp. 1960, 2010.

[42] M. Guerquin-Kern, L. Lejeune, K. P. Pruessmann, and M. Unser, "Realistic analytical phantoms for parallel magnetic resonance imaging," IEEE Trans. Med. Imag., vol. 31, no. 3, pp. 626-636, 2012.

[43] N.-k. Chen and A. M. Wyrwicz, "Correction for epi distortions using multi-echo gradient-echo imaging," Magn. Reson. Med., vol. 41, no. 6, pp. 1206-1213, 1999.

[44] G. T. Luk-Pat and D. G. Nishimura, "Reducing off-resonance distortion by echo-time interpolation," Magn. Reson. Med., vol. 45, no. 2, pp. 269-276, 2001

[45] H. Moriguchi, J. S. Lewin, and J. L. Duerk, "Dixon techniques in spiral trajectories with off-resonance correction: a new approach for fat signal suppression without spatial-spectral rf pulses," Magn. Reson. Med., vol. 50, no. 5, pp. 915-924, 2003.

[46] J. Medič and S. Tomažič, "Off-resonance frequency filtered magnetic resonance imaging," Magn. Reson. Imaging, vol. 28, no. 4, pp. 527536, 2010.

[47] D. Ma, V. Gulani et al., "Magnetic resonance fingerprinting," Nature, vol. 495, no. 7440, p. 187, 2013.

[48] M. Davies, G. Puy, P. Vandergheynst, and Y. Wiaux, "A compressed sensing framework for magnetic resonance fingerprinting," SIAM J. Imaging Sci., vol. 7, no. 4, pp. 2623-2656, 2014.

[49] G. Mazor, L. Weizman, A. Tal, and Y. C. Eldar, "Low-rank magnetic resonance fingerprinting," Medical physics, vol. 45, no. 9, pp. 40664084, 2018.

[50] Y. Jiang, D. Ma, N. Seiberlich, V. Gulani, and M. A. Griswold, "MR fingerprinting using fast imaging with steady state precession (fisp) with spiral readout," Magn. Reson. Med., vol. 74, no. 6, pp. 1621-1631, 2015.

[51] Z. Wang, H. Li, Q. Zhang, J. Yuan, and X. Wang, "Magnetic resonance fingerprinting with compressed sensing and distance metric learning," Neurocomputing, vol. 174, pp. 560-570, 2016.

[52] G. Mazor, L. Weizman, A. Tal, and Y. C. Eldar, "Low rank magnetic resonance fingerprinting," in IEEE Annual Int. Conf. EMBC. IEEE, 2016, pp. 439-442

[53] C. Liao, B. Bilgic et al., " $3 \mathrm{~d}$ MR fingerprinting with accelerated stack-of-spirals and hybrid sliding-window and grappa reconstruction," Neuroimage, vol. 162, pp. 13-22, 2017.

[54] X. Cao, C. Liao et al., "Robust sliding-window reconstruction for accelerating the acquisition of MR fingerprinting," Magn. Reson. Med., vol. 78, no. 4, pp. 1579-1588, 2017.

[55] J. Vymazal, A. Righini et al., "T1 and $\mathrm{T} 2$ in the brain of healthy subjects, patients with parkinson disease, and patients with multiple system atrophy: relation to iron content," Radiology, vol. 211, no. 2, pp. 489-495, 1999 . 